\title{
Long-segment percutaneous fixation: various rather than proper indications
}

\author{
Bernhard Meyer
}

Received: 11 February 2011 / Accepted: 14 February 2011 /Published online: 3 March 2011

(C) Springer-Verlag 2011

I was invited to comment on the manuscript by Blondel et al. [1] describing their experience with percutaneous spinal long-segment fixation with two commercially available systems.

I believe that these systems have their (narrow) spectrum of indications and therefore this should be communicated to the community to propagate their use in a sensible manner. However, I have doubts that this article serves that purpose. On the contrary, I am convinced that it does just the opposite, by providing arguments to the opponents against their use.

I apologize to the authors beforehand for being harsh in this editorial, but I feel strongly that the message of this article-which is printed in an influential peer-reviewed journal-needs to be put in the proper perspective very clearly for various reasons.

The very obvious fact is that a heterogeneous population comprising a small number of subjects treated for various indications (as the authors state themselves) with two different systems is studied retrospectively with a short follow-up and undefined outcome variables. This alone forbids any valid conclusion beyond a mere descriptive statement that percutaneous spinal long-segment fixation is technically feasible.

This, however, is not my main problem, and I could accept publication despite these severe shortcomings.

The most important weakness of this report relates to indications and surgical technique. I challenge the management of every case the authors show (or

\section{B. Meyer $(\bowtie)$}

Department of Neurosurgery, Technical University of Munich,

Klinikum rechts der Isar, Ismaninger Str. 22,

81675 Munich, Germany

e-mail: Bernhard.meyer@1rz.tum.de describe). I very well accept that there is usually more than one way to treat a patient, but there is nowadays a broad consensus on the mere principles of treatment for certain spinal disorders.

Exactly these basic principles were violated just for the sake of using a new tool, and this is a completely wrong message.

I dare to say that if most of the cases were shown during a major spine congress, the vast majority in the audience would agree.

On the verge of being rude, I am also willing to predict that opinion leaders in orthopedic spinal surgery will use this article as an argument why neurosurgeons should stick to their own business.

I will give several examples to illustrate this.

The trauma cases The case in figure 3 was a Type A3 fracture in a 50-year-old. To perform kyphoplasty in a nonosteoporotic fracture is not an accepted indication (the authors state to have done this in six of their seven cases of non-osteoporotic fractures!). If the patient has too many comorbidities, then treat him conservatively in the first place. Otherwise a proper anterior reconstruction of the disc space (plus canal clearance, which is not shown on the postoperative picture!) is mandatory and easily achievable in every patient. If you think of "preserving" motion segments, then the alternative is additional screws in the index level and a scheduled hardware removal. Both of these were not done. The authors state in their table that Type A1 and 2 fractures were treated. Most people would not consider these fracture types surgical candidates, let alone with a long-segment fixation.

The degenerative scoliosis cases One of the problems with long-segment percutaneous systems is the difficulty in proper 
deformity correction. Three principles are violated as can be seen on the postoperative $\mathrm{x}$-rays. Sagittal deformity correction is far more important than coronal. The authors have achieved a slight correction in the coronal plane, but left the patient with a flat back and without sagittal balance. Add to this that the rods are too long and restrict motion in two segments above the construct. Add to this that this then ends in the thoracolumbar junction zone and the chances are very high that this will end in a failure. By the way, I am unable to see an anterior support on this picture. If a final result is to be appreciated by the reader, the pictures after the anterior reconstruction need to be shown.

The tumor cases The case illustrated in figure 1 is also very debatable. Why have the authors included so many motion segments in this construct? A two-level fixation above and below L1 including vertebroplasty of it would have been enough. The upper thoracic level (if symptomatic) would have been well treated with cement augmentation alone. This would also have avoided that the rods were again way too long, which restricts motion on two more cranial levels.
The infectious case It is well known in the last 5-7 years that long autologous strut grafts, i.e., iliac crest spanning two disc spaces, has a pseudarthrosis rate of more than $50 \%$. Consensus is nowadays to rather use distractible titanium cages if this is the case, since titanium alloys have excellent properties for use in infectious environments.

To conclude, I would encourage the readers to remain skeptical when new tools are offered with the label of being less invasive (="better"), without a clear rationale and a proper indication for their use being given.

Conflicts of interest None.

\section{References}

1. Blondel B, Fuentes S, Tropiano P, Roche PH, Mete'llus P, Dufour H (2011) Systems for long-segment percutaneous fixation: technical feasibility for various indications. Acta Neurochir 